Agron. Mesoam. 29(2):375-387. Mayo-agosto, 2018

ISSN 2215-3608, doi:10.15517/ma.v29i2.29546

http://www.revistas.ucr.ac.cr/index.php/agromeso

\title{
Degradabilidad ruminal de la materia orgánica de variedades de Cenchrus purpureus tolerantes a sequía ${ }^{1}$
}

\author{
Ruminal degradability of organic matter of varieties of drought tolerant \\ Cenchrus purpureus
}

José Leonardo Ledea-Rodríguez ${ }^{2}$, Jorge Valentín Ray-Ramírez², Orestes La-O-León ${ }^{3}$, Juan José Reyes-Pérez,

\section{Resumen}

La principal limitante de las gramíneas tropicales es su alto contenido de carbohidratos estructurales, los cuales, determinan el aprovechamiento del pasto por el animal. Cuando los pastos o forrajes crecen en ecosistemas adversos se presentan cambios importantes en la degradabilidad ruminal de los compuestos de interés. El objetivo fue caracterizar, bajo diferentes edades de rebrote, la degradabilidad ruminal in situ de la materia orgánica de diferentes variedades de Cenchrus purpureus mejorados genéticamente para tolerar ambientes secos. Se emplearon tres variedades (CT-601, CT-603 y CT-605) tolerantes a sequía, en diferentes edades de rebrote (60, 80, 100 y 120 días). Se utilizaron dos vacas criollas de $400 \pm 50 \mathrm{~kg}$ de peso vivo, fistuladas, las bolsas se introdujeron en el rumen por 0 , 4, 6, 8, 12, 24, 48 y 72 horas, la estimación de la degradación ruminal se realizó a partir del ajuste de los datos a la ecuación exponencial $\left.(\mathrm{a}+\mathrm{b})^{*}\left(1-\mathrm{e}^{-\mathrm{-}^{*} \mathrm{t}}\right)\right)$. El mejor comportamiento en la degradabilidad ruminal in situ de la fracción potencialmente degradable $(\mathrm{a}+\mathrm{b})$ de hojas, se observó a los ochenta días de edad, mientras que la afectación de la dinámica de degradación por el efecto de la edad de rebrote, fue común para hojas y tallos. La fracción a presentó porcentajes de degradación que no superaron el 10\% para hojas y tallos; sin embargo, la degradación de b superó el $71 \%$ para hojas y el $30 \%$ para tallos. Las nuevas variedades mostraron un patrón de fermentación ruminal cercano a $50 \%$, característico de las gramíneas tropicales.

Palabras claves: rumiantes, Pennisetum, clima árido, gramíneas, digestión ruminal.

\begin{abstract}
The main limitation of tropical grasses is their high content of structural carbohydrates, which determine the use of grass by animals. When pastures or forages grow in adverse ecosystems there are important changes in the ruminal degradability of the compounds of interest. The objective was to characterize, at different ages of regrowth, the in situ ruminal degradability of the organic matter of different varieties of Cenchrus purpureus genetically improved to tolerate dry environments. Three drought tolerant varieties (CT-601, CT-603, and CT-605) were taken at different regrowth ages $(60,80,100$, and 120 days). Two fistulated Creole cows of $400 \pm 50 \mathrm{~kg}$ of live weight were used.
\end{abstract}

1 Recibido: 5 de junio, 2017. Aceptado: 23 de noviembre, 2017. Este trabajo formó parte del proyecto "Rehabilitación de sistemas pastoriles de la región oriental cubana", incluido en el programa nacional de producción de alimento animal de la República de Cuba, Cuba.

2 Instituto de Investigaciones Agropecuarias “Jorge Dimitrov," Estación Experimental de Pastos y Forrajes, km 101⁄2, Carretera Bayamo - Tunas. Bayamo, Granma, Cuba. ledea1017@gmail.com; jvray2011@gmail.com

3 Instituto de Ciencia Animal (ICA), Apartado Postal 24, San José de las Lajas, Mayabeque, Cuba. olao@ica.co.cu

4 Universidad Técnica de Cotopaxi. Extensión La Maná. Av. Los Almendros y Pujilí, Edificio Universitario, La Maná, Ecuador.

5 Universidad Técnica Estatal de Quevedo. Av. Walter Andrade. Km 1 1⁄2 vía a Santo Domingo. Quevedo, Los Ríos, Ecuador. jjreyesp1981@ gmail.com 
The bags were introduced in rumen for $0,4,6,8,12,24,48$, and 72 hours, the estimation of the rumen degradation was made fitting the data to the exponential equation $(\mathrm{a}+\mathrm{b}) *(1-\mathrm{e}(-\mathrm{c} * \mathrm{t}))$. The best performance in in situ ruminal degradability of the potentially degradable fraction $(a+b)$ of leaves was observed at the age of eighty days, while the effect of the degradation dynamics due to the effect of regrowth age was common for leaves and stems. Fraction a degradation values did not exceed $10 \%$ for leaves and stems; however, the degradation of b showed values that exceeded $71 \%$ for leaves and $30 \%$ for stems. The new varieties showed a ruminal fermentation pattern close to $50 \%$, characteristic of tropical grasses.

Keywords: ruminants, Pennisetum, arid climate, grasses, rumen digestion.

\section{Introducción}

Las gramíneas tropicales poseen como potencialidad, acumular gran cantidad de biomasa por unidad de superficie en un intervalo de tiempo determinado. Son caracterizadas por presentar un rápido crecimiento y ser plantas muy eficientes en el aprovechamiento de la energía solar, por presentar los senderos $\mathrm{C}_{3}$ y $\mathrm{C}_{4}$ y desarrollarse además, en ecosistemas de altas temperaturas, donde estas influyen en la velocidad de crecimiento cuando las precipitaciones no son una limitante.

El régimen pluviométrico determina el crecimiento y productividad de los cultivos, al constituir la materia prima de los procesos metabólicos y mantener la integridad de las membranas celulares (Fortes, 2014). En la última década, y de forma acelerada por efecto del cambio climático, el régimen y distribución de las precipitaciones ha cambiado de forma radical, estableciéndose una favorable disponibilidad de alimentos cuando son abundantes, pero también un marcado déficit de pasturas cuando las lluvias son escasas.

En Cuba, principalmente en el Valle del Cauto, ubicado en la región oriental del país, se desarrolla el 90\% de la ganadería, el efecto de las precipitaciones dispone, al menos seis meses en el año, un déficit alimentario por el efecto de la sequía, que se traduce en cuantiosas pérdidas económicas, debido a que, los cultivos naturalizados e introducidos no toleran el intenso período de pocas precipitaciones, que se extiende desde noviembre hasta abril.

Para reducir el efecto de la falta de precipitaciones en la productividad animal y mantener cultivos en el campo en el período de pocas precipitaciones, en la década del 2000 comenzó el programa de obtención de cultivares de Cenchrus purpureus a partir del CT-115. Como resultado de este programa, se obtuvieron clones tolerantes a la sequía. Al realizar la caracterización molecular de estas plantas, mediante la determinación electroforética de más de cinco sistemas isoenzimáticos (Herrera, 2000), demostró que eran variedades, esto se reafirmó al mantener en estas variedades sus características iniciales durante más de cinco generaciones.

En los estudios agronómicos realizados en la región oriental de Cuba, las nuevas variedades tolerantes a la sequía (CT-601, CT-603 y CT-605), superaron a su progenitor en la respuesta agronómica (Almaguer, 2012; Arias, 2012; Ray et al., 2016). La evaluación de estas variedades se realizó en ecosistemas degradados por la intensa sequía estacional, y se les podría considerar como una opción para el incremento del germoplasma que se utiliza en el Valle del Cauto e introducirse en ecosistemas donde las precipitaciones son una limitante; sin embargo, es necesario conocer que impacto tienen las fracciones que mayormente son consumidas por los animales en la degradabilidad ruminal, en diferentes edades de rebrote.

Por el característico rápido crecimiento de las gramíneas, ocurre una rápida transformación a nivel de la pared celular en diferentes estadios de crecimiento que atentan contra el aprovechamiento de los carbohidratos estructurales. Al extenderse la edad del cultivo, ocurren cambios fisiológicos inherentes a la planta que, conllevan a transformaciones morfo-histológicas que, estimulan la relación e interrelación de los carbohidratos, mediante enlaces covalentes, prevaleciendo entre los enlaces los puentes de hidrógeno y puentes firulatos originados a partir del ácido firúlico que está contenido en la pared celular de las células vegetales (Cherney, 2000). Dichos enlaces 
se combinan con terminales libres de otros compuestos estructurales que no son carbohidratos, como la lignina, deprimiendo el valor nutritivo por constituir una barrera para la acción enzimática microbiana, lo cual constituye una de las razones por las cuales las gramíneas tropicales no alcanzan el 50\% de degradación de la materia seca (MS) y orgánica (MO) (Cáceres, 1985; Ramírez, 2010), y se acentúan por el efecto de las variables climáticas de forma independiente y su combinación (Ledea, 2016).

Cerca de las dos terceras partes de la materia orgánica (MO) contenida en los tejidos es digerida por los microorganismos ruminales, responsables de degradar el alimento para que el animal se sirva de los productos de sus procesos metabólicos (Cáceres, 1985). La digestibilidad de la MO se consideró la mejor expresión del valor nutritivo desde el punto de vista energético de los forrajes, que puede ser tan alta como el $80 \%$ en gramíneas tiernas y descender hasta el 50\% o menos en los forrajes maduros, en correspondencia con el comportamiento de las variables climáticas y hábito de crecimiento de la planta (Cáceres, 1985).

El objetivo del presente estudio fue caracterizar, bajo diferentes edades de rebrote, la degradabilidad ruminal in situ de la materia orgánica de diferentes variedades de Cenchrus purpureus mejorados genéticamente para tolerar ambientes secos.

\section{Materiales y métodos}

\section{Localidad y clima}

El estudio se llevó a cabo en la Estación Experimental de Pastos y Forrajes (EEPF), perteneciente al Instituto de Investigaciones Agropecuarias (IIA) "Jorge Dimitrov", localizado en la provincia Granma en la llanura del Cauto, a 10,5 km de la ciudad de Bayamo, Cuba. El clima donde está ubicada la estación se clasifica como tropical relativamente húmedo (Barranco y Díaz, 1989). En el área de estudio la temperatura media del aire varía entre $24,2^{\circ} \mathrm{C}$ en época seca y hasta $27,7^{\circ} \mathrm{C}$ en época de lluvia, con valores máximos de $28,6^{\circ} \mathrm{C}$ y $32,8^{\circ} \mathrm{C}$, respectivamente (Díaz, 2007). En 2013 y 2014 precipitaron 783 y 924,1 mm, respectivamente. Del total de milímetros precipitados, el 94,3\% y $69,8 \%$ se distribuyeron en el período lluvioso (mayo-octubre) para ambos años, con períodos intensamente secos en la estación de pocas precipitaciones (noviembre-abril).

\section{Material vegetal utilizado}

Se emplearon tres variedades de Cenchrus purpureus (CT-601, CT-603 y CT-605), establecidas en parcelas de $\left(200 \mathrm{~m}^{2}\right)$ en la EEPF. Las variedades fueron obtenidas a partir de cultivos de tejidos de conos apicales del CT115 (Herrera, 2000), y fueron las que superaron a su progenitor en las evaluaciones agronómicas desarrolladas en ecosistemas secos del Valle del Cauto. Para el establecimiento de los bancos, las semillas fueron proporcionadas por el departamento de Pastos y Forrajes del Instituto de Ciencia Animal, Mayabeque, Cuba.

\section{Procedimiento experimental}

A las variedades en estudio se les fertilizó con materia orgánica de origen bovino a razón de 25 t/ha, durante el corte de establecimiento. Para ello, se taró una carretilla y se llenó con la cantidad correspondiente en función del área y dosis de fertilizante a utilizar. Durante el proceso de llenado de la carretilla se contaron las palas con las que se alcanzaba el peso deseado, luego fue trasladada al surco y se aplicó a lo largo del mismo. El pesaje de la 
carretilla con la materia orgánica se realizó solo una vez, luego con el número de palas que llegaba al peso deseado, se procedió a aplicar la fertilización al resto de los surcos.

Al momento de tomar las muestras, en cada surco, de forma intercalada se habían distribuido las edades a evaluar (60, 80, 100 y 120 días), estaban fraccionados por subparcelas de 4,50 m lineales con cuatro repeticiones y $1 \mathrm{~m}$ de efecto de borde al comienzo y final de cada surco, producto de un estudio agronómico que se estaba realizando al unísono. Aprovechando este diseño, se procedió a tomar de cada repetición cinco plantas en cada edad de rebrote, para un total de veinte plantas por muestreo, y un total de cuatro muestreos para los 60 días, tres para 80 y 100 días, y dos para 120 días que abarcaron el período lluvioso y poco lluvioso.

Para facilitar la maniobrabilidad de las plantas, se fraccionó el tallo y se separaron las hojas con tijera de acero inoxidable, luego se tomaron $300 \mathrm{~g}$ en base verde y seguidamente, fueron homogenizadas en base seca para la determinación de la composición química y degradabilidad ruminal in situ. Posteriormente, se sometieron a secado en estufa de aire forzado a $100{ }^{\circ} \mathrm{C}$ durante una hora y luego a $60{ }^{\circ} \mathrm{C}$ hasta peso constante, según la metodología propuesta por Herrera et al. (2003), reduciéndolo en partículas de $2 \mathrm{~mm}$ en molino de cuchillas para la degradabilidad ruminal in situ (100 g), y $200 \mathrm{~g}$ a $1 \mathrm{~mm}$, para la determinación de la composición química. Este procedimiento se realizó en cada edad de rebrote, luego se conformó un "pool" de las muestras colectadas en ambos períodos climáticos (lluvioso y poco lluvioso), que resultó en una muestra única para cada edad de cada variedad para las determinaciones de composición química y degradabilidad ruminal in situ.

\section{Composición química}

El análisis de las muestras de las variedades se realizó en hojas y tallos. Se determinó el contenido de MO, proteína bruta $(\mathrm{PB})$ y cenizas $(\mathrm{Cz})$, de acuerdo con las técnicas descritas por AOAC (2016). En el Cuadro 1 se describe la composición química de las variedades en diferentes edades de rebrote.

Cuadro 1. Composición química en base seca de hojas y tallos de variedades de Cenchrus purpureus con diferentes edades de rebrote, cultivadas en la Estación Experimental de Pastos y Forrajes del Instituto de Investigaciones Agropecuarias "Jorge Dimitrov", provincia Granma, Cuba. 2013-2014.

Table 1. Chemical composition on dry base of leaves and stems of Cenchrus purpureus varieties at different regrowth ages, grown at the Experimental Pasture and Forage Station of the Institute of Agricultural Research "Jorge Dimitrov", Granma province, Cuba. 2013-2014.

\begin{tabular}{|c|c|c|c|c|c|c|c|c|c|c|c|c|}
\hline \multirow{4}{*}{$\begin{array}{l}\text { Constituyentes } \\
\text { químicos (\%) }\end{array}$} & \multicolumn{12}{|c|}{ Edad de rebrote } \\
\hline & \multicolumn{3}{|c|}{60} & \multicolumn{3}{|c|}{80} & \multicolumn{3}{|c|}{100} & \multicolumn{3}{|c|}{120} \\
\hline & \multicolumn{12}{|c|}{ Variedades (CT) } \\
\hline & 601 & 603 & 605 & 601 & 603 & 605 & 601 & 603 & 605 & 601 & 603 & 605 \\
\hline & \multicolumn{12}{|c|}{ Hojas } \\
\hline $\mathrm{PB}(\mathrm{N} \times 6,25)$ & 8,2 & 11 & 9,2 & 8 & 7,7 & 8,1 & 7,9 & 7,2 & 8,2 & 7,1 & 7 & 7,8 \\
\hline MO & 82,9 & 82 & 76,9 & 83,2 & 82,2 & 82,1 & 84,3 & 83,1 & 82,6 & 85,7 & 83,4 & 86,5 \\
\hline \multirow[t]{2}{*}{$\mathrm{Cz}$} & 14,2 & 16,7 & 13,4 & 15,6 & 16,8 & 17,3 & 16,7 & 17,5 & 17,8 & 17,0 & 17,9 & 23,02 \\
\hline & \multicolumn{12}{|c|}{ Tallos } \\
\hline $\mathrm{PB}(\mathrm{N} \times 6,25)$ & 2,7 & 3,5 & 3,5 & 3,1 & 3,9 & 4 & 3,3 & 3,7 & 5,3 & 5 & 5,3 & 5,8 \\
\hline MO & 89,5 & 87,8 & 88,01 & 91,4 & 90,1 & 89 & 89,7 & 90,3 & 87,3 & 94,1 & 93,2 & 93,2 \\
\hline $\mathrm{Cz}$ & 10,4 & 12,1 & 11,9 & 8,5 & 9,8 & 10,9 & 10,2 & 9,6 & 12,6 & 5,8 & 6,7 & 6,7 \\
\hline
\end{tabular}

PB: proteína bruta, MO: materia orgánica, Cz: cenizas, CT: cultivo de tejidos / PB: crude protein, OM: organic matter, Cz: Ash, CT: tissue culture. 


\section{Degradabilidad ruminal in situ}

Para esta evaluación se utilizaron dos vacas criollas canuladas en rumen (400 $\pm 50 \mathrm{~kg}$ de peso vivo) que, recibían forraje de gramíneas (OM-22, Megathyrsus maximum y Cenchrus ciliaris) ad libitum, con libre acceso al agua y sales minerales. Se pesaron $5 \mathrm{~g}$ de muestras por bolsa de fibra sintética de polyester, después de haber constatado la uniformidad de las dimensiones internas mediante una regla milimetrada, tamaños de poro y cantidad de poros ( $\mathrm{cm}^{2}$ medidos), y promediados con la ayuda de un microscopio electrónico con cámara acoplada, ordenador y software Image Driving. Las dimensiones fueron uniformes para todas las bolsas de $14,0 \mathrm{~cm}$ de largo x $8,5 \mathrm{~cm}$ de ancho de dimensiones internas y $48 \mu \mathrm{m}$ de porosidad y 1044 poros $/ \mathrm{cm}^{2}$. Luego las bolsas fueron lavadas y secadas en estufa a $60{ }^{\circ} \mathrm{C}$ durante una hora, posteriormente, se pesaron en una balanza analítica de $0,001 \mathrm{~g}$ de precisión, donde se determinó la tara a cada una de las bolsas incluidas en el experimento.

Las bolsas se agruparon por variedad para introducirlas en el rumen, se duplicaron para cada horario de muestreo y animal. Las bolsas se introdujeron para la fermentación ruminal todas juntas y se extrajeron a las 0,4 , $6,8,12,24,48$ y 72 horas. Cada bolsa se lavó a mano con agua corriente hasta que, el agua salió transparente una vez extraídas del rumen; posteriormente, se depositaron en bandejas de aluminio y se secaron en una estufa a 60 ${ }^{\circ} \mathrm{C}$ durante 72 horas, luego se transfirieron a una desecadora durante $30 \mathrm{~min}$ y se procedió al pesado, la diferencia entre el peso inicial de la muestra colocada en las bolsas de nylon y el peso del residuo después de la incubación ruminal, se utilizó para determinar la MO degradada en el rumen, después de que fue incinerada por $24 \mathrm{~h}$ a $550{ }^{\circ} \mathrm{C}$. La solubilidad a la hora cero se obtuvo según las sugerencias de Pedraza et al. (2007), introduciendo dos bolsas con cada alimento en rumen hasta que penetrara completamente el líquido ruminal al interior de las mismas, después se trataron según la metodología de evaluación de la degradabilidad ruminal in situ.

La estimación de la degradación ruminal se realizó mediante el proceso interactivo del algoritmo de MARQUARDT, con ayuda del procedimiento para modelos no lineares PROC NLIN del software Statgraphics Centurion versión XVII. Para la estimación de las características degradativas, se utilizó el modelo exponencial propuesto por Ørskov y Mc Donald (1979), asumiendo que la curva de degradación de la MS en el tiempo sigue un proceso cinético de primer orden que, se describe de la forma:
$\mathrm{P}=\mathrm{A}$
$(a+b) *\left(1-e^{\wedge}(-c * t)\right)$
para $\mathrm{t} 0=0$
t>to

Donde:

P: degradación ruminal. Es la degradación ruminal del indicador evaluado en el tiempo " $\mathrm{t}$ " de permanencia en el rumen $(\%)$.

a: intercepto (fracción soluble si el tiempo no es limitante) \%.

b: fracción degradable, si el tiempo no es limitante (t) $\%$.

c: tasa de degradación de la fracción (b) \%/h.

t: tiempo de incubación (h).

a+b: potencial de degradación (\%).

A: fracción rápidamente soluble. Se obtiene de varias maneras. En este estudio se determinó mediante la introducción en el rumen de la bolsa, para que penetrara el líquido ruminal al interior de la misma, se extrajo de inmediato, luego de que se sumergiera completamente en el líquido ruminal, y se prosiguió el procedimiento descrito para la degradabilidad in sacco. 


\section{Resultados}

Cuando se analizaron los parámetros que componen la degradabilidad ruminal in situ de la MO en hojas, se observó el paulatino deterioro de la degradación en función del incremento de la edad de rebrote.

Los parámetros de degradación ruminal in situ de la materia orgánica contenida en las hojas de las tres variedades y los valores de la fracción soluble, variaron entre 5 y 15,6\%, y a partir de los cien días disminuyeron su degradación en más de un 50\%, caracterizándose este proceso por una gran variabilidad (Cuadro 2, Figura 1).

Cuadro 2. Degradabilidad ruminal in situ de la materia orgánica en hojas de nuevas variedades de Cenchrus purpureus tolerantes a la sequía, en diferentes edades de corte, cultivadas en la Estación Experimental de Pastos y Forrajes del Instituto de Investigaciones Agropecuarias “Jorge Dimitrov", provincia Granma, Cuba. 2013-2014.

Table 2. In situ ruminal degradability of organic matter in leaves of new drought-tolerant varieties of Cenchrus purpureus, at different cutting ages, grown at the Experimental Pasture and Forage Station of the Institute of Agricultural Research "Jorge Dimitrov", Granma province, Cuba. 2013-2014.

\begin{tabular}{|c|c|c|c|c|c|}
\hline Edad en días & Variedades & Modelos & $a+b(\%)$ & $\mathbf{R}^{2}$ & $\operatorname{EEE}(\%)$ \\
\hline \multirow{3}{*}{60} & 601 & $\mathrm{Y}=5,00+45,00\left(1-\exp ^{-0,062 x \operatorname{tiempo}}\right)$ & 50,01 & 90,4 & 17,8 \\
\hline & 603 & $\mathrm{Y}=12,17+45,17\left(1-\mathrm{exp}^{-0,046 x \text { tiempo }}\right)$ & 57,34 & 85,1 & 27,4 \\
\hline & 605 & $\mathrm{Y}=15,65+46,05\left(1-\exp ^{-0,052 x \text { tiempo }}\right)$ & 61,70 & 87 & 25,8 \\
\hline \multirow{3}{*}{80} & 601 & $\mathrm{Y}=8,71+56,71\left(1-\exp ^{-0,064 x \mathrm{tiempo}}\right)$ & 65,43 & 85,1 & 25,8 \\
\hline & 603 & $\mathrm{Y}=10,96+50,96\left(1-\exp ^{-0,092 x \text { tiempo }}\right)$ & 61,93 & 87,6 & 21,4 \\
\hline & 605 & $\mathrm{Y}=11,54+47,54\left(1-\right.$ exp $\left.^{-0,055 x \text { tiempo }}\right)$ & 59,09 & 95,1 & 15,01 \\
\hline \multirow{3}{*}{100} & 601 & $\mathrm{Y}=5,13+26,13\left(1-\exp ^{-0,071 \text { xiempo }}\right)$ & 31,27 & 85,6 & 29,2 \\
\hline & 603 & $\mathrm{Y}=6,29+40,29\left(1-\exp ^{-0,062 x \text { tiempo }}\right)$ & 46,59 & 85,9 & 19,1 \\
\hline & 605 & $\mathrm{Y}=6,33+59,33\left(1-\exp ^{-0,050 x \text { xiempo }}\right)$ & 65,67 & 85,6 & 29,2 \\
\hline \multirow{3}{*}{120} & 601 & $\mathrm{Y}=9,56+32,56\left(1-\exp ^{-0,050 x i e m p o}\right)$ & 42,12 & 85,6 & 29,2 \\
\hline & 603 & $\mathrm{Y}=7,60+30,60\left(1-\exp ^{-0,145 x \text { tiempo }}\right)$ & 38,20 & 94,2 & 6,52 \\
\hline & 605 & $\mathrm{Y}=6,91+37,91\left(1-\exp ^{-0,051 x \text { tiempo }}\right)$ & 44,82 & 85,3 & 18,9 \\
\hline
\end{tabular}

$(a+b)$ : degradación potencial calculadas según modelo $a+b *\left(1-e^{\wedge\left(-c^{*} t\right)}\right)$, a: fracción soluble, b: fracción insoluble pero potencialmente degradable, c: velocidad de degradación, $\mathrm{R}^{2}$ : coeficiente de determinación, EEE: Error estándar de la estimación / (a+b): potential degradation calculated by model $a+b^{*}\left(1-e^{\wedge\left(-c^{*} t\right)}\right)$, a: soluble fraction, $b$ : insoluble fraction but potentially degradable, $c$ : degradation rate, $\mathrm{R}^{2}$ : determination coefficient, EEE: estimate standard error.

Para los tallos, la degradabilidad (Cuadro 3, Figura 2) se mostró mucho más deprimida que la degradación de las hojas. En este órgano es característico la presencia de células con paredes celulares muy gruesas, con desarrolladas paredes primarias y secundarias denominadas esclereidas, y en muchos casos estas están desprovistas de citoplasto.

\section{Discusión}

La degradabilidad ruminal in situ de la MO en hojas de C.purpureus fue variable (Cuadro 2 y Figura 1), al respecto, Ledea (2016) señaló que, los constantes cambios en la estructura y composición química, estimulados principalmente 

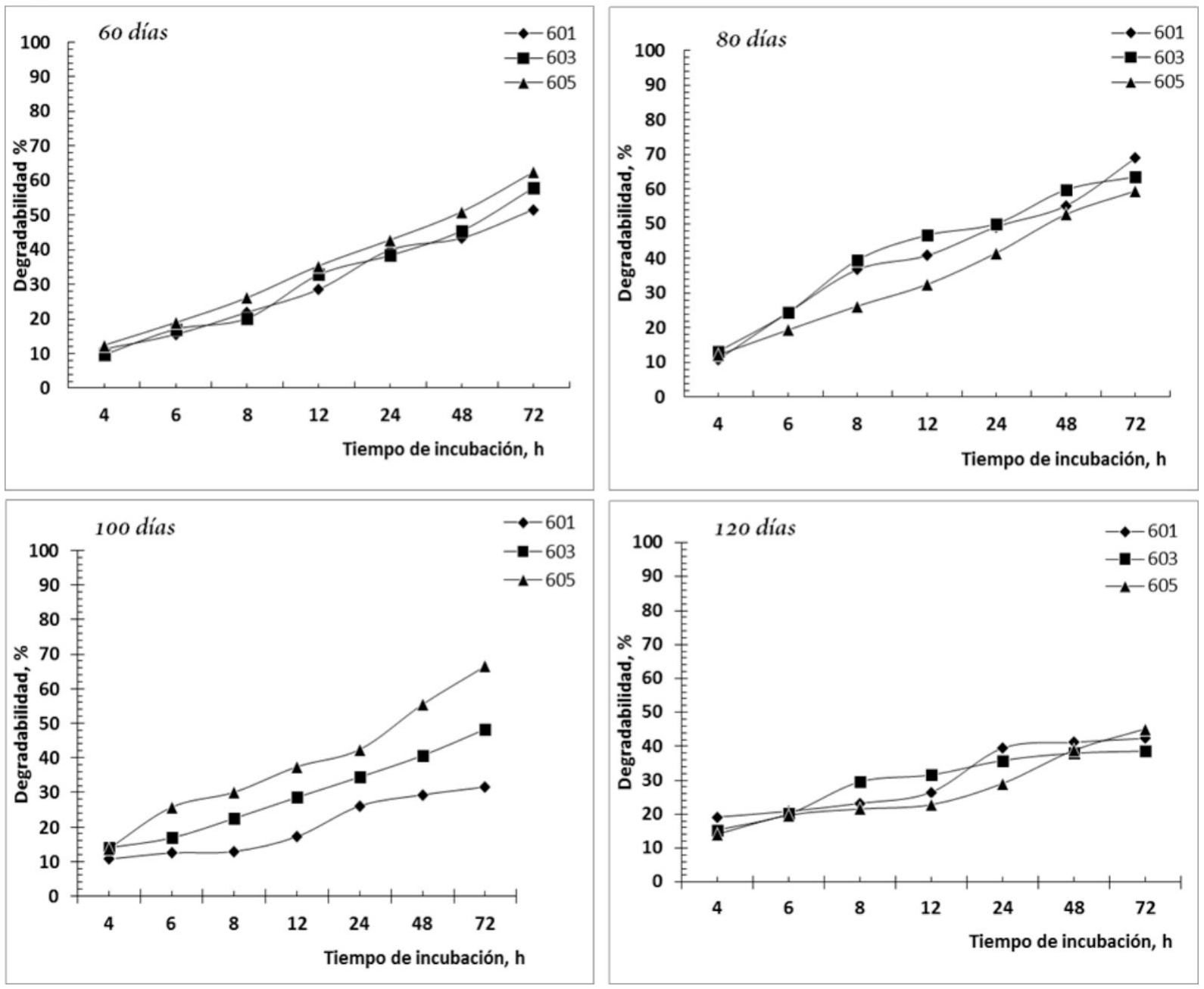

Figura 1. Dinámica de la degradabilidad ruminal in situ de la materia orgánica de hojas de tres variedades de Cenchrus purpureus, a las edades de 60, 80, 100 y 120 días de rebrote, cultivadas en la Estación Experimental de Pastos y Forrajes del Instituto de Investigaciones Agropecuarias "Jorge Dimitrov", provincia Granma, Cuba. 2013-2014.

Figure 1. Dynamics of in situ ruminal degradability of organic matter of leaves of new varieties of Cenchrus purpureus at 60, 80, 100 and 120 days of regrowth, grown at the Experimental Pasture and Forage Station of the Institute of Agricultural Research "Jorge Dimitrov", Granma province, Cuba. 2013-2014.

por la interacción de la edad de rebrote con la estación climática, limitan su utilización por la microflora ruminal, Ruiz y De-Arriba (1987) refirieron que, de la concentración de MO en la pared celular (35- 80\%), solo se degrada entre el 65-67\% de forma establecida, estos valores superaron a los obtenidos en este estudio, estimuladas las diferencias por las condiciones climáticas en las que se desarrollaron los cultivos características de la región del trópico en la que se ubica Cuba, donde los rayos solares inciden perpendicularmente y con ello, se estimula la síntesis de carbohidratos estructurales, sin embargo, los valores mostrados en el Cuadro 2, superaron los indicados por Edwards et al. (2012) en Brachiaria erecta, debido a que, estos autores utilizaron a la planta íntegra, y en este estudio solo se contemplaron las hojas, órganos donde se concentra la mayor cantidad de carbohidratos solubles. 
Cuadro 3. Degradabilidad ruminal in situ de la materia orgánica en tallos de tres variedades de Cenchrus purpureus tolerantes a la sequía en diferentes edades de rebrote, cultivadas en la Estación Experimental de Pastos y Forrajes del Instituto de Investigaciones Agropecuarias "Jorge Dimitrov", provincia Granma, Cuba. 2013-2014.

Table 3. In situ ruminal degradability of organic matter of stems of three drought-tolerant varieties of Cenchrus purpureus at different ages of regrowth, grown at the Experimental Pasture and Forage Station of the Institute of Agricultural Research, "Jorge Dimitrov", Granma province, Cuba. 2013-2014.

\begin{tabular}{|c|c|c|c|c|c|}
\hline Edad en días & Variedades & Modelos & $a+b(\%)$ & $\mathbf{R}^{2}$ & EEE $(\%)$ \\
\hline \multirow{3}{*}{60} & 601 & $\mathrm{Y}=7,32+20,3\left(1-\exp ^{-0,1036 x \text { tiempo }}\right)$ & 27,64 & 90,4 & 17,8 \\
\hline & 603 & $\mathrm{Y}=4,54+51,54\left(1-\exp ^{-0,051 \text { xtiempo }}\right)$ & 56,09 & 85,1 & 27,4 \\
\hline & 605 & $\mathrm{Y}=4,57+23,57\left(1-\exp ^{-0,075 x \operatorname{tiempo}}\right)$ & 28,15 & 87 & 25,8 \\
\hline \multirow{3}{*}{80} & 601 & $\mathrm{Y}=7,46+39,46\left(1-\exp ^{-0,043 x \text { tiempo }}\right)$ & 46,93 & 85,1 & 25,8 \\
\hline & 603 & $Y=13,99+31,99\left(1-\exp ^{-0,059 x \text { tiempo }}\right)$ & 45,98 & 87,6 & 21,4 \\
\hline & 605 & $\mathrm{Y}=8,88+39,88\left(1-\right.$ exp $\left.^{-0,050 x \operatorname{xiempo}}\right)$ & 48,77 & 95,1 & 15,01 \\
\hline \multirow{3}{*}{100} & 601 & $\mathrm{Y}=5,51+39,51\left(1-\mathrm{exp}^{-0,061 \times \text { xiempo }}\right)$ & 45,02 & 85,6 & 29,2 \\
\hline & 603 & $Y=10,25+37,25\left(1-\exp ^{-0.067 x \text { xiempo }}\right)$ & 47,50 & 85,9 & 19,1 \\
\hline & 605 & $\mathrm{Y}=6,74+29,74\left(1-\exp ^{-0,027 \times x i e m p o}\right)$ & 36,48 & 85,6 & 29,2 \\
\hline \multirow{3}{*}{120} & 601 & $\mathrm{Y}=6,96+27,96\left(1-\exp ^{-0,045 x \text { tiempo }}\right)$ & 34,93 & 85,6 & 29,2 \\
\hline & 603 & $\mathrm{Y}=8,19+40,19\left(1-\exp ^{-0,034 x \operatorname{tiempo}}\right)$ & 48,39 & 94,2 & 6,52 \\
\hline & 605 & $\mathrm{Y}=9,49+36,49\left(1-\exp ^{-0,042 x \operatorname{tiempo}}\right)$ & 45,98 & 85,3 & 18,9 \\
\hline
\end{tabular}

$(a+b)$ : degradación potencial calculadas según modelo $a+b^{*}\left(1-e^{\wedge\left(-c^{*} t\right)}\right)$, a: fracción soluble, b:fracción insoluble pero potencialmente degradable, c:velocidad de degradación, $\mathrm{R}^{2}$ : coeficiente de determinación, EEE: error estándar de la estimación / (a+b): potential degradation calculated by model $\mathrm{a}+\mathrm{b}^{*}\left(1-\mathrm{e}^{\wedge\left(-\mathrm{c}^{*} \mathrm{t}\right)}\right)$, a: soluble fraction, $\mathrm{b}$ : insoluble fraction but potentially degradable, c: degradation rate, $\mathrm{R}^{2}$ : determination coefficient, EEE: standard error of estimation.

El efecto negativo de la edad de rebrote en la degradación de la MO contenida en los tejidos de las hojas (Cuadro 2, Figura 1), se debe a que la edad de rebrote estimula el desarrollo de complejas relaciones e interrelaciones que se establecen entre los constituyentes de la pared celular. De estas complejas relaciones, se obtenían degradaciones de la materia orgánica inferiores al 50\% según lo indicado por Cáceres (1985) y Geissman y Neukom (1971), ya habían referido que los enlaces mediante los cuales se unen los monosacáridos y polisacáridos son covalentes del tipo $\beta 1-4$, al igual que los que unen a los arabinoxilanos y la lignina (Ishii, 1991), con ramificaciones desde $\beta$ 1-2 hasta $\beta$ 1-4 y $\alpha$ 1-3 hasta $\alpha$ 1-5 (Pérez-Infante, 2013). La prevalencia de estos enlaces limita la degradación de los constituyentes de la pared celular, ya que el animal cuenta con muy pocos microorganismos plurienzimáticos en relación con la biota que habita el rumen, presentando la gran mayoría de los microbios ruminales enzimas con acción homogénea sobre los enlaces $\beta$ 1-4. Algunas bacterias y, de forma mayoritaria, hongos, presentan acción plurienzimática, sin embargo, el pH determina la acción de los mismos (6-7), y cuando la dieta es rica en carbohidratos el pH redunda entre 4 y 5 que es donde se desarrollan satisfactoriamente las bacterias (Galindo et al., 2014). En el presente estudio no se monitoreó la variación del pH, sin embargo, es uno de los aspectos que determinan el accionar de los microorganismos ruminales y que pudo estar manifiesto por las bajas degradaciones de los parámetros de degradación, si hubieran intervenido otros microorganismos que no fueran las bacterias se hubiera logrado una mayor degradación de la pared celular por la acción plurienzimática, independientemente de las limitaciones que predisponen la distribución molecular de los monosacáridos y polisacáridos que estructuran la pared celular. 


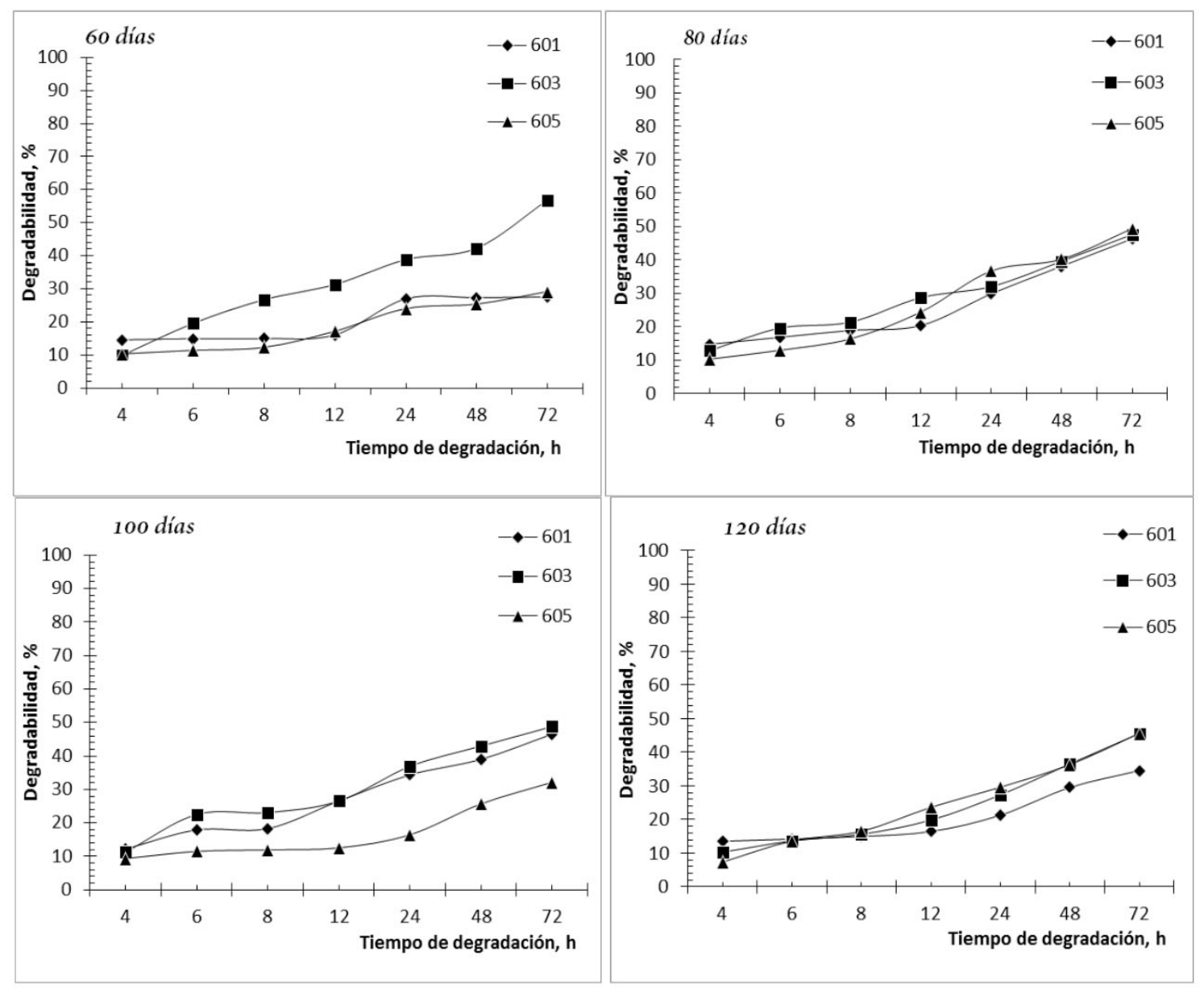

Figura 2. Dinámica de la degradabilidad ruminal in situ de la materia orgánica de tallos de tres variedades de Cenchrus purpureus a las edades de 60, 80, 100 y 120 días de rebrote, cultivadas en la Estación Experimental de Pastos y Forrajes del Instituto de Investigaciones Agropecuarias "Jorge Dimitrov”, provincia Granma, Cuba. 2013-2014.

Figure 2. Dynamics of in situ ruminal degradability of organic matter of stems of new varieties of Cenchrus purpureus at $60,80,100$ and 120 days of regrowth, grown at the Experimental Pasture and Forage Station of the Institute of Agricultural Research "Jorge Dimitrov", Granma province, Cuba. 2013-2014.

Si se tienen en cuenta los criterios expuestos, se justifica que, con el incremento de la edad de rebrote, prevalezcan los carbohidratos estructurales y con ello, la reducción del acceso microbiano a la parte orgánica de la pared y lumen celular, al ser estos dos factores (edad de rebrote e incremento de los constituyentes de la pared celular) directamente proporcionales. Por lo tanto, la organización de los constituyentes de la pared celular predispone el acceso de los microorganismos y la acción enzimática sobre los sustratos específicos (Chesson et al., 1983), y esto se refleja en la degradabilidad de las diferentes fracciones (Pedraza, 2000; Valenciaga, 2007; Beachumin et al., 2008; Valenciaga et al., 2009; Fortes, 2014; Pedraza, 2014; Caro y Dihigo, 2015) y tiempo de retención en el rumen, que varió entre 4,6 y 14,5\%/hora.

La degradación de la fracción b también disminuyó con el incremento de la edad de rebrote, y varió entre 26,1 y $56,7 \%$. El factor que pudo haber influido en este comportamiento de la fracción insoluble pero potencialmente degradable, fueron los bajos tenores de proteínas que presentaron estas variedades, Minson (1980) señaló que, 
valores inferiores al 7\% deprimen la degradabilidad ruminal por no permitir una utilización eficiente de los carbohidratos estructurales por parte de las bacterias, estas se ven limitadas por la disponibilidad de esqueletos carbonados de los aminoácidos que son utilizados para la síntesis de proteína microbiana de alto valor biológico y permiten su multiplicación en el rumen, y con ello, la efectividad de la degradación de la porción orgánica.

Los bajos porcentajes de la fracción a y b, determinaron que no se lograra una degradación potencial que alcanzara valores que variaron entre 65-67\%, referidos por Ruiz y De-Arriba (1987) como aceptables, sino que estuvieron entre 31,2 y 61,9\%. La depresión en la degradación de la MO es característica de las pasturas tropicales (Ramírez et al., 2002). Este comportamiento degradativo de las fracciones de la MO se reflejó en su dinámica (Figura 1), en este sentido Bannink y Tamminga (1993) sugirieron que, la mejor manera de expresar la producción microbiana era relacionarla con la degradación de la materia orgánica. En función de este criterio, se observó que la degradación en el tiempo con énfasis en la masa microbiana fue variable. Los comportamientos para todas las edades fueron ascendentes, y hasta los ochenta días se alcanzaron degradaciones superiores al 50\%, y picos de despegue a partir de las doce horas aproximadamente, esto se debió a que los primeros microorganismos en actuar son las bacterias (Galindo et al., 2014), luego se suman los hongos y los protozoos que potencian la degradación al complementar la relaciones interespecíficas y de mutualismo que están establecidas entre los microbios ruminales. Estos resultados fueron superiores a los reportados por La-O et al. (2009) en la planta íntegra de Cordia alba (Jacq), colectadas en el oriente de Cuba, e inferiores a los comunicados por Valenciaga et al. (2009), cuando evaluaron el efecto de la edad de rebrote en la degradabilidad in vitro del CT-115, y relacionaron la elevada degradabilidad de la MO con el alto contenido de nitrógeno y carbohidratos solubles presentes en las hojas de CT-115, característica que fue señalada por Herrera et al. (2003), Valenciaga (2007) y La-O et al. (2009), compuestos que constituyen la materia prima para el desarrollo bacteriano principalmente (Galindo et al., 2014).

La depresión de la degradabilidad de los tallos está asociada, según Ledea (2016), a la distribución de los tejidos y con ellos, sus estructuras químicas, que varían dentro de un mismo órgano, diferentes órganos y partes de la planta, criterio que coincidió con el expuesto por Pérez-Infante (2013). La celulosa es uno de los compuestos mayoritarios en los tallos de las gramíneas (Ledea, 2016), y es el principal en tallos del género Cenchrus (Valenciaga, 2007; Ramírez, 2010; Pérez-Infante, 2013). La celulosa se une al resto de los carbohidratos estructurales mediante puentes de hidrógeno, que se caracterizan por presentar fuertes enlaces y gran cantidad de energía entre ellos, dependiendo de la fuerza e intensidad de la carga y el ángulo de enlace entre los átomos (Fortes, 2014); además de las fuerzas de Van der Waals (Cárdenas et al., 2016), que impiden de forma muy marcada la degradación del monosacárido por la acción enzimática. Esta molécula incrementa la polimerización en función de la edad de la planta, y se incrementa también el número de grupos hidroxilos libres correspondientes a los átomos de carbono 2, 3 y 6, que son los responsables de la estructura supra molar (fibra vegetal), otorgándole a la fibra un carácter hidrófobo, que limitan el acceso de las bacterias y con ello, la degradación de la fracción orgánica principalmente.

Se obtuvo para la fracción soluble degradaciones entre 5,5 y 13,9\%, y en la fracción $b 20,3$ y $51,4 \%$, para una degradabilidad efectiva que se comportó entre 27,6 y $48,7 \%$, con velocidades de degradación de 2,7-10,3\%/hora. El valor nutritivo, según Pérez-Infante (2013), no hace más que reflejar las características histológicas y morfológicas de las plantas, haciendo mención como principales factores la especie de pasto, el estado de desarrollo, la edad, la fertilización, el uso o no del riego, entre otros; pero sin considerar el comportamiento molecular de los monosacáridos y polisacáridos constituyentes de las paredes celulares, y las relaciones que se establecen entre ellos (Weiss y Demarquilly, 1970).

Las asociaciones y modificaciones que se establecen entre los carbohidratos estructurales, estimulados por diferentes fuentes externas y otras de origen biológico de las que se ha hecho alusión en diferentes ocasiones, limitan la degradación enzimática, y son una de las causas por las cuales difícilmente las gramíneas tropicales sobrepasan el 50\% de degradabilidad (Minson, 1980; Barahona-Rosales y Sánchez-Pinzón, 2005).

Lo expuesto acerca del efecto de la edad de rebrote con respecto a la degradabilidad, no coincide con el 
criterio de Cáceres (1985), quien refirió que no siempre el valor nutritivo de un pasto se reduce conforme avanza la edad, sino que intervienen otros factores; sin embargo, no lo demostró en el estudio que desarrolló. Este criterio coincidió con el expuesto por Benavides (1977), quien tras evaluar al pasto kikuyo (Pennisetum clandestinum) bajo diferentes edades de corte, concluyó que la madurez en este tipo de pasto no presenta gran influencia en el valor nutritivo, debido seguramente, a las características climáticas de la región donde se desarrolló su experimento. Las condiciones climáticas en que se desarrollaron las plantas en estudio, pudieron estimular el fortalecimiento de los enlaces covalentes que se establecen entre los polímeros de la pared celular, la lignina y los carbohidratos estructurales, con tendencia a incrementar la polimerización y ramificación de los constituyentes estructurales (Hernández, 1999), lo que estimula y acelera las modificaciones en la organización molecular de las membranas de la célula y en su pared celular (Fortes, 2014).

La cinética de degradación de los tallos reflejó las limitaciones de la masa microbiana para degradar los compuestos orgánicos (Figura 1), los comportamientos fueron similares, y se denotó el efecto de la edad de rebrote en la depresión de la degradabilidad ruminal. El criterio de Valenciaga et al. (2009), estuvo dado por las asociaciones lignina-hemicelulosa que se establecen en este tipo de órgano (de sostén y conducción) que dificultan el acceso de los microorganismos, así como el adecuado acoplamiento de las enzimas microbianas con los sustratos específicos (Capanema et al., 2005), lo que se demuestra en el tiempo que les toma a los microorganismos poder acceder a la materia orgánica contenida en las paredes celulares y degradarla (Figura 2). En las gramíneas jóvenes, aunque se encuentran con bajos contenidos de fibra, el principal constituyente de esta es la celulosa que se encuentra en su forma más pura (Cáceres, 1985), y puede constituir una barrera para la acción microbiana por el estado de pureza principalmente, que le dispone enlaces ricos en pirol que acidifica el medio donde se encuentre, variando el $\mathrm{pH}$ ruminal y con él, los microorganismos que pueden accionar, acceder y multiplicarse (Galindo et al., 2014).

\section{Conclusiones}

Las nuevas variedades en estudio mostraron un patrón de fermentación ruminal con degradaciones cercanas al 50\% para hojas y más deprimido para los tallos, característico de las gramíneas tropicales. La edad de rebrote afectó la cinética de degradación en hojas y tallos, en función del incremento de la misma.

\section{Literatura Citada}

Almaguer, R. 2012. Evaluación del establecimiento de variedades de Pennisetum purpureum resistentes a la sequía en condiciones de premontaña. Tesis Lic., Universidad de Granma, Bayamo, CUB.

AOAC (Official Methods of Analysis). 2016. Official methods of analysis of AOAC International. 20 ${ }^{\text {th }}$ ed. AOAC International, Rockville, MD, USA.

Arias, R. 2012. Frecuencias de corte en cultivares promisorios de Pennisetum purpureum resistentes a la sequía con riego y fertilización orgánica. Tesis MSc., Universidad de Granma, CUB.

Bannink, A., and S. Tamminga. 1993. Rumen function. In: J.M. Forbes, and J. France, editors, Quantitative aspects of ruminant digestion and metabolism. CAB Int., Wageningen, HOL. p. 263-290.

Barranco, G., y L.R. Díaz. 1989. Clima. En: Instituto de Geografía, editor, Nuevo Atlas Nacional de Cuba. VI. 1. 2. Instituto Geográfico, Verde olivo, CUB. p. 15-20.

Barahona-Rosales, R., y S. Sánchez-Pinzón. 2005. Limitaciones físicas y químicas de la digestibilidad de pastos tropicales y estrategias para aumentarla. Rev. Corpoica 6(1):69-82. doi:10.21930/rcta.vol6_num1_art:39 
Beachumin, R.A., M.O. Kreuser, F.P. O`Mora, and T.A. McAliester. 2008. Nutritional management for enteric methane abatement: A review. Austr. J. Exp. Agric. 48:21-27. doi:10.1071/EA07199

Benavides, E. 1977. Valor nutritivo del pasto kikuyo. Rev. ICA 12(4):638.

Cáceres, O. 1985. Estudio de los principales factores que afectan el valor nutritivo de las gramíneas forrajeras tropicales en Cuba. Tesis Dr., Centro Universitario "Camilo Cienfuegos”, Matanzas, CUB.

Capanema, E.A., M.Y. Balakshin, and J.F. Dadla. 2005. A comprehensive approach for quantitative lignin characterization by NMR spectroscopy. J. Agric. Food Chem. 52:1850-1860. doi:10.1021/jf035282b

Caro, Y.C., y L.D. Dihigo. 2015. Comportamiento productivo de conejos alimentados con dietas que incluían harina integral de dólico y mucuna. Rev. Unellez Cienc. Tecnol. 30:29-35.

Cárdenas, A.L., L.J. Bautista, L.J. Zegarra, R. Ramos, O.E. Gómez, y J.S. Barreto. 2016. Degradabilidad in situ de la materia seca y proteína cruda de las hojas y peciolo del Pisonay (Erythrina falcata). Rev. Vet. Perú. 27(1):39-44. doi:10.15381/ rivep.v27i1.11461

Cherney, I.R. 2000. Characterization of forages by chemical analysis. In: D.I. Owen et al., editors, Forage evaluation in ruminant nutrition. CAB International, GBR. p. 281-300.

Chesson, A., A.H. Gordon, and J.A. Lomax. 1983. Substituent groups linked by alkali-labile bonds to arabinose and xylose residues of legume, grass and cereal straw cell walls and their fate during digestion by rumen organisms. J. Sci. Food Agric. 34:1330-1340. doi:10.1002/jsfa.2740341204

Díaz, D. 2007. Evaluación agronómica de nuevas variedades Pennisetum purpureum en condiciones de sequía el Valle del Cauto. Tesis MSc., Universidad de Matanzas, Matanzas, CUB.

Edwards, A., V. Mlambo, C.H.O. Pallo, W.G Garcia, and M.D. Diptee. 2012. In vitro ruminal fermentation parameters of tanner grass (Brachiaria erecta) supplemented with leaves from three forage trees. Liv. Res. Rural Dev. 24(6). http://www.lrrd. org/lrrd24/6/edwa24102.htm

Fortes, D. 2014. Comportamiento de algunos indicadores morfofisiológicos y de calidad de Pennisetum purpureum vc. CT-115 utilizado como banco de biomasa. Tesis Dr., Instituto de Ciencia Animal, Mayabeque, CUB.

Galindo, J., N. González, Y. Marrero, y A. Sosa. 2014. Efecto del follaje de plantas tropicales en el control de la producción de metano y la población de protozoos ruminales in vitro. Rev. Cub. Cienc. Agric. 48:359-364.

Geissman, T., and H. Neukom. 1971. Vernetzung von Phenolcarbonsaureestern von Polysacchariden durch oxydative phenolicshe kupplung. Helv Chim Acta 54:1108-1112.

Hernández, A.1999. Nueva versión de la clasificación genética de los suelos de Cuba. AGRINFOR-MINAG, La Habana, CUB.

Herrera, R.S. 2000. Obtención de plántulas de Pennisetum purpureum con resistencia a la sequía y salinidad mediante técnicas biotecnológicas. CITMA-ICA, La Habana, CUB.

Herrera, R.S., Z. Chaple, A.M. Cruz, A. Romero, y M. García. 2003. Obtención de plántulas de Pennisetum purpureum resistentes a la sequía y salinidad. Rev. Cub. Cienc. Agríc. 37:189-191.

Ishii, T. 1991. Isolation and characterization of a diferuloyl arabinoxylan hexasaccharide from bamboo shoot cell-walls. Carbohydr. Res. 219:15-22. doi:10.1016/0008-6215(91)89039-I

La-O, O., M.A. Solís, O. Ruiz, H. González, Y. Castillo, E. Gutiérrez, A. Muro, C. Arzola, C. Rodríguez, y J.G. Cairo. 2009. Potencial fermentativo in vitro y degradabilidad ruminal in situ de materia seca y materia orgánica de Uvita, Cordia alba (Jacq), en ecosistemas del oriente de Cuba. Rev. Cub. Cienc. Agríc. 43:39-44. 
Ledea, J.L. 2016. Caracterización químico nutritiva de nuevas variedades de Cenchrus purpureus tolerantes a la sequía en el Valle del Cauto. Tesis MSc., Universidad de Granma, CUB.

Minson, D.J. 1980. Nutritional differences between tropical and temperate pasture. In: F.H.W. Morley, editor, Grazing animals. Elsevier, Amsterdam, HOL. p. 143-157.

Ørskov, E.R., and I. McDonald. 1979. The estimation of protein degradability in the rumen from incubation measurements weighted according to rate of passage. J. Agric. Sci. 92:499-503. doi:10.1017/S0021859600063048

Pedraza, C.D. 2014. Evaluación de la actividad enzimática de aislamientos microbianos celulolíticos y lignolíticos, y su aplicación en la degradación de tamo de arroz (Oryza sativa). Tesis MSc., Universidad Nacional de Colombia, COL.

Pedraza, R. 2000. Valoración nutritiva del follaje de Gliricidia sepium (Jacq.) Kunt ex Walp. y su efecto en el ambiente ruminal. Tesis Dr., Instituto de Ciencia Animal, La Habana, CUB.

Pedraza, R.M., T. Sánchez, E. Green, R. Mayes, y E.R. Ørskov. 2007. Efecto de incubar el forraje seco o fresco en las características de su producción de gas in vitro. [CD], ICA, La Habana, CUB.

Pérez-Infante, F. 2013. Ganadería eficiente. Asociación Cubana de Producción Animal, La Habana, CUB.

Ramírez, J.L. 2010. Rendimiento y calidad de cinco gramíneas en el Valle del Cauto. Tesis Dr., Instituto de Ciencia Animal, Mayabeque, CUB.

Ramírez, R., R.G. Ramírez, y F. López. 2002. Factores estructurales de la pared celular del forraje que afectan su digestibilidad. Cienc. UANL 5:180-189.

Ray, J., R.S. Herrera, D. Benítez, D. Díaz, and R. Arias. 2016. Multivariate analysis of the agronomic performance and forage quality of new clones of Pennisetum purpureum drought tolerant in Valle del Cauto, Cuba. Cuban J. Agric. Sci. 50:639-648.

Ruiz, R., y J. De-Arriba. 1987. Digestión ruminal de carbohidratos y absorción de AGV. En: Sánchez et al., editores, Bioquímica nutricional. Tomo I. Verde olivo, La Habana, CUB. p. 143-177.

Valenciaga, D. 2007. Caracterización química y estructural de las paredes celulares de Pennisetum purpureum vc. CUBA CT-115 y su digestibilidad ruminal en búfalos de río (Bubalis bubalus). Tesis Dr., Instituto de Ciencia Animal, Mayabeque, CUB.

Valenciaga, D., B. Chongo, S.R. Herrera, V. Torres, A. Oramas, y M. Herrera. 2009. Efecto de la edad de rebrote en la digestibilidad in vitro de la materia seca de Pennisetum purpureum vc. CUBA-CT 115. Rev. Cub. Cienc. Agríc. 43:81-84.

Weiss, P., et C. Demarquilly. 1970. Valeur alimnetaire des fourrages verts. Fourrages 42:3-22. 